\title{
Medidas de biossegurança no enfrentamento da COVID-19
}

\author{
Biosafety measures facing COVID-19 \\ Medidas de bioseguridad frente al COVID-19
}

Recebido: 27/10/2021 | Revisado: 05/11/2021 | Aceito: 12/11/2021 | Publicado: 21/11/2021

Vinicius Urquiza da Nóbrega Porto
ORCID: https:///orcid.org/0000-0001-7734-8003
Faculdade de Ciências Médicas da Paraíba, Brasil
Email: urquizavinicius2002@ @otmail.com
Ana Beatriz Oliveira Galvão
ORCID: https://orcid.org/0000-0002-5050-6371
Faculdade de Ciências Médicas da Paraíba, Brasil
E-mail: anabeatrizoliveira141 @gmail.com
Wanderson Gomes Veloso
ORCID: https://orcid.org/0000-0002-8529-3345
Faculdade de Ciências Médicas da Paraíba, Brasil
E-mail: wanderson.b215@gmail.com
Nieara Saad Rached de Souza
ORCID: https://orcid.org/0000-0001-6067-2341
Faculdade de Ciências Médicas da Paraíba, Brasil
E-mail: niearasaad4@ gmail.com
Iasmin Pordeus Coura Urtiga
ORCID: https://orcid.org/0000-0002-6825-8089
Faculdade de Ciências Médicas da Paraíba, Brasil
E-mail: Iasminpordeus.c @ gmail.com
Fabiana Medeiros de Brito
ORCID: https://orcid.org/0000-0003-3824-0168
Faculdade de Ciências Médicas da Paraíba, Brasil
E-mail: fabianabrito_@ hotmail.com

\begin{abstract}
Resumo
Objetivo: Caracterizar a produção científica acerca das medidas de biossegurança utilizadas no enfrentamento da COVID-19. Métodos: Trata-se de uma revisão integrativa, realizada no período de abril de 2021 com o intuito de promover a sustentação teórica com base na literatura. Os estudos foram selecionados nas bases de dados LILACS, MEDLINE e SciELO, emergindo ao final uma amostra de 15 artigos. Resultados e Discussão: da análise dos dados emergiram duas categorias temáticas: I - "Medidas de Biossegurança adotadas na pandemia de COVID-19"; e II "Impactos da COVID-19 na Biossegurança e na qualidade de vida dos profissionais da saúde". Considerações finais: As medidas de biossegurança são essenciais para a contenção do coronavírus, pois protege o profissional, os pacientes e o restante da população. Apesar das problemáticas que envolvem o tema, como a falta de políticas públicas eficientes para a contenção do vírus, os profissionais enfrentam cargas de trabalho elevadas para atender os infectados da melhor maneira possível, mesmo quando há desfalque de leitos e de equipamentos adequados.
\end{abstract}

Palavras-chave: Biossegurança; COVID-19; Profissionais de saúde.

\begin{abstract}
Objective: To characterize the scientific production about biosafety measures used to fight COVID-19. Methods: This is an integrative review, carried out in April 2021 with the aim of promoting theoretical support based on the literature. The studies were selected from the LILACS, MEDLINE and SciELO databases, resulting in a sample of 15 articles. Results and Discussion: from the data analysis, two thematic categories emerged: I - "Biosafety measures adopted in the COVID-19 pandemic"; and II - "Impacts of COVID-19 on Biosafety and on the quality of life of health professionals". Final considerations: Biosecurity measures are essential to contain the coronavirus, as it protects professionals, patients and the rest of the population. Despite the problems surrounding the topic, such as the lack of efficient public policies to contain the virus, professionals face high workloads to attend to the infected in the best possible way, even when there is a shortage of beds and adequate equipment.
\end{abstract}

Keywords: Biosafety; COVID-19; Health professionals.

\section{Resumen}

Objetivo: Caracterizar la producción científica sobre las medidas de bioseguridad utilizadas para combatir el COVID19. Métodos: Se trata de una revisión integradora, realizada en abril de 2021 con el objetivo de promover el soporte teórico con base en la literatura. Los estudios fueron seleccionados de las bases de datos LILACS, MEDLINE y SciELO, 
dando como resultado una muestra de 15 artículos. Resultados y Discusión: del análisis de datos surgieron dos categorías temáticas: I - "Medidas de bioseguridad adoptadas en la pandemia de COVID-19"; y II - "Impactos del COVID-19 en la Bioseguridad y en la calidad de vida de los profesionales de la salud". Consideraciones finales: Las medidas de bioseguridad son fundamentales para contener el coronavirus, ya que protege a los profesionales, a los pacientes y al resto de la población. A pesar de los problemas que rodean el tema, como la falta de políticas públicas eficientes para contener el virus, los profesionales enfrentan altas cargas de trabajo para atender a los infectados de la mejor manera posible, incluso cuando hay escasez de camas y equipamiento adecuado.

Palabras clave: Bioseguridad; COVID-19; Profesionales de la salud.

\section{Introdução}

A Organização Mundial da Saúde (OMS) decretou em 2020 o estado de pandemia do novo coronavírus (SARS-CoV2), iniciado na província de Hubei, na China, doença denominada de COVID-19 (Campos \& Leitão, 2021). Este decreto alertou sobre a alta capacidade de disseminação do vírus e a necessidade de estratégias para a contenção do mesmo por meio de isolamento social e medidas de higiene (Campos \& Leitão, 2021).

Segundo o relatório atualizado do Johns Hopkins Coronavírus Resource Center (2020), 131.612.552 pessoas foram infectadas pelo novo coronavírus no mundo, dentre essas 2.856.790 evoluíram para óbito até 5 de abril de 2021. Já no Brasil, foram 12.984.956 casos e 331.433 óbitos até essa mesma data.

A COVID-19 se manifesta de diferentes modos, podendo apresentar sintomas semelhantes aos de outras doenças respiratórias ou complicações, a ponto de necessitar de suporte ventilatório, ou até mesmo evoluir para óbito. Sua sintomatologia substancial é: febre, tosse seca, fadiga, mialgia, anosmia, hiposmia e ageusia, podendo evoluir para dispneia ou até Síndrome Respiratória Aguda Grave (SRAG) (Gautier \& Ravussin, 2020).

Frente a referida pandemia, têm-se discutido cada vez mais sobre a necessidade de medidas que contribuam para o controle da prevenção desta doença. A biossegurança diz respeito à proteção da vida, envolvendo quem desenvolve o trabalho, a quem ele é dirigido (usuário/doente) e o espaço institucional, social e ambiental onde ele ocorre. As medidas de biossegurança criam barreiras entre os profissionais e os agentes danosos, além da disponibilização e o emprego correto e adequado de materiais e equipamentos contribuem para a proteção coletiva (Ribeiro, Pires \& Scherer, 2016).

No âmbito dos serviços de saúde, como a exemplo hospitais e unidades básicas de saúde, as medidas de biossegurança são implementadas por normas, regulamentações e protocolos de segurança, que devem ser respeitados pelos profissionais envolvidos, evitando-se assim exposição a determinados tipos de riscos, como a exposição a agentes biológicos, capazes de promover agravos à saúde humana e ambiental (Teixeira \& Valle, 2010).

Dentre tais medidas, destaca-se a utilização dos Equipamentos de Proteção Individual (EPI’s) e, para que esses sejam realmente eficazes, é fundamental que os profissionais de saúde sejam previamente treinados quanto à paramentação e à desparamentação, bem como informados quanto às medidas de propagação e mitigação da doença (Gallasch, Cunha, Pereira, \& Silva-Junior, 2020). Por conseguinte, a correta utilização destes EPI’s reduzem os riscos de infecção e promovem maior segurança em cada profissional.

Nessa perspectiva, medidas de proteção e segurança se tornam indispensáveis, visto o alto grau de infectividade e virulência do SARS-CoV-2, além da alta capacidade de transmissibilidade, sendo disseminado através gotículas respiratórias, contato e secreções, além de gerar casos letais, sobretudo em grupos de risco (Soares et al., 2020). A despeito disso, ações de prevenção devem ser tomadas para evitar a exposição e o despreparo, sobretudo, em ambiente hospitalar, garantindo a segurança de todos os envolvidos (Ribeiro, Oliveira, Silva, \& Souza, 2020).

Para garantir ainda mais a segurança dos profissionais e pacientes vítimas de COVID-19, algumas práticas se tornam substanciais, como o isolamento do paciente infectado, com redução e controle do número de profissionais, devidamente paramentados, nestes ambientes (Ribeiro, Oliveira, Silva, \& Souza, 2020; Soares et al., 2020). Ademais, os profissionais da 
saúde devem utilizar as precauções padrão em casos suspeitos, como lavar as mãos antes e depois do contato, além do uso e distribuição obrigatórias dos EPI’s, requeridos pela NR 32, como capotes, máscaras do tipo PFF2 (N95), óculos e roupas protetoras, ocorrendo treinamento prévio quanto ao uso destes equipamentos, além de realizar escalas de turno para a redução da exposição e sobrecarga dos profissionais, afastando aqueles de maior risco e vulnerabilidade (Ribeiro, Oliveira, Silva, \& Souza, 2020; Soares et al., 2020).

Dessa forma, surge a necessidade de enfoque acerca do conhecimento de boas técnicas no manejo do paciente com COVID-19, atrelado ao uso correto dos EPI's e das noções de biossegurança, além da necessidade de notificar os acidentes ocupacionais, para promover uma mudança eficiente dessa realidade. Ressalta-se ainda que tais medidas básicas, implicam na redução de riscos ocupacionais e na qualidade de vida dos profissionais de saúde, grupo que contabiliza cerca de 180 mil óbitos desde o início da pandemia (OMS, 2021). Assim, estudos como este podem facilitar e orientar medidas para auxiliar nas boas técnicas no cuidado à COVID-19

Por conseguinte, considera-se uma temática de grande relevância, visto que a COVID-19 é uma doença descoberta recentemente e os impactos provocados por ela irão refletir muito na sociedade tanto atualmente como no futuro. Nesse contexto, o objetivo do presente estudo foi caracterizar a produção científica acerca das medidas de biossegurança utilizadas no enfrentamento da COVID-19.

\section{Metodologia}

Trata-se de uma revisão integrativa da literatura com fins de identificar, analisar e sintetizar resultados independentes sobre o mesmo assunto, proporcionando assim, uma compreensão ampla e completa do fenômeno analisado, e contribuindo para uma possível repercussão benéfica na qualidade dos cuidados oferecidos ao usuário, como também na construção do pensamento crítico que a nossa prática diária necessita (Souza, Silva, \& Carvalho, 2010).

Diante do exposto percebe-se que a Revisão Integrativa da Literatura se constitui como um instrumento da Prática Baseada em Evidências (PBE). No entanto, para operacionalização dessa revisão, utilizaram-se as seguintes etapas: elaboração da pergunta norteadora; busca ou amostragem na literatura; coleta de dados mediante critérios de inclusão e exclusão; análise crítica dos estudos incluídos; discussão dos resultados e apresentação da revisão integrativa (Souza, Silva, \& Carvalho, 2010). Respeitando os referidos critérios, foi elaborada a seguinte pergunta norteadora da pesquisa: Mediante as evidências científicas, quais as medidas de biossegurança utilizadas pelos profissionais da saúde no enfrentamento da COVID-19?

A busca dos dados foi realizada durante o mês abril de 2021, por meio da Biblioteca Virtual em Saúde (BVS), delimitando as seguintes bases de dados: Literatura Latino-americana e do Caribe em Ciências da Saúde (LILACS), Medical Literature Analysis and Retrieval System Online (MEDLINE) e Scientific Electronic Library Online (SciELO). Os Descritores em Ciências da Saúde (Decs) selecionados para responder à questão foram: Biossegurança; COVID-19; Profissionais de Saúde. A seguir, procedeu-se à busca dos artigos, utilizando-se os três descritores ligados pelo conector AND. Foram adotados os seguintes critérios de inclusão para a seleção dos artigos: aqueles encontrados no portal da BVS, nos idiomas (português, espanhol e inglês), disponibilizados na íntegra, publicados entre o período de junho de 2020 a março de 2021, que abordassem a temática no título e/ou resumo e/ou palavra-chave. Foram excluídos estudos que não atendessem a questão norteadora e aos critérios de inclusão mencionados, além de artigos que não demonstram adequadamente o referencial teórico e metodológico e/ou rigor científico e ético.

Foram obtidos inicialmente, a partir da pesquisa nas bases de dados, utilizando os descritores, 28 artigos. Após a aplicação dos filtros, ocorrendo em seguida a leitura minuciosa de cada título, resumo e artigo na íntegra, para selecionar artigos que se relacionavam com o objetivo do estudo, 15 artigos foram escolhidos. Suas informações organizadas em um instrumento de coleta de dados, contendo: título do artigo, ano de publicação, base de dados, objetivo do trabalho, periódico. 
Posteriormente, os artigos selecionados foram lidos na íntegra e analisados, de acordo com o instrumento de coleta de dados validado por Ursi e Galvão (2006). Na sequência, os dados obtidos por meio do material reunido (oriundo dos trechos extraídos das publicações) foram organizados em planilhas, com agrupamento das informações, de acordo com a relevância e a equivalência das categorias temáticas que configuram o objetivo principal do estudo. Depois dessa planificação e da organização, procedeu-se à análise temática dos referidos dados.

\section{Resultados e Discussão}

O estudo foi constituído por 15 publicações que versaram sobre a temática "Medidas de biossegurança no enfrentamento da COVID-19", conforme caracterização explicitada no Quadro 1 a seguir:

Quadro 1 - Distribuição dos estudos segundo o título, a base de dados, o periódico, o ano de publicação e idioma - 2020 a 2021 - João Pessoa (PB), 2021

\begin{tabular}{|c|c|c|c|}
\hline Autor (es) & Título do estudo & $\begin{array}{c}\text { Base de } \\
\text { dados }\end{array}$ & Idioma \\
\hline $\begin{array}{l}\text { Silveira, M. G. S. S., } \\
\text { et al. }\end{array}$ & $\begin{array}{l}\text { Changes in dental practice in times of COVID-19: review and } \\
\text { recommendations for dental health care }\end{array}$ & LILACS & $\begin{array}{c}\text { Inglês } \\
2021\end{array}$ \\
\hline $\begin{array}{l}\text { Vicente, K. M. S., } \\
\text { Silva, B. M., \& } \\
\text { Barbosa, D. N. }\end{array}$ & $\begin{array}{l}\text { Diretrizes de biossegurança para o atendimento odontológico durante a } \\
\text { pandemia do COVID-19: revisão de literatura }\end{array}$ & $\begin{array}{l}\text { LILACS, } \\
\text { BBO }\end{array}$ & $\begin{array}{c}\text { Português } \\
2020\end{array}$ \\
\hline $\begin{array}{l}\text { Godoi, A. P. N., } \\
\text { Bernardes, G. C. S., } \\
\text { Novais, R. L. R., } \\
\text { Nogueira, L. S., \& } \\
\text { Pinheiro, M. B. }\end{array}$ & $\begin{array}{l}\text { Focus on Health professionals during the SARS-COV-2 pandemic- } \\
\text { Literature Review }\end{array}$ & LILACS & $\begin{array}{c}\text { Inglês } \\
2020\end{array}$ \\
\hline Misra, V., et al. & $\begin{array}{l}\text { Guidelines for various laboratory sections in view of COVID-19: } \\
\text { Recommendations from the Indian Association of Pathologists and } \\
\text { Microbiologists }\end{array}$ & MEDLINE & $\begin{array}{c}\text { Inglês } \\
2020\end{array}$ \\
\hline $\begin{array}{l}\text { Quadros, A., } \\
\text { Fernandes, M. T. C., } \\
\text { Araujo, B. R., \& } \\
\text { Caregnato, R. C. A. }\end{array}$ & $\begin{array}{l}\text { Desafios da Enfermagem Brasileira no Combate da COVID-19: uma } \\
\text { reflexão }\end{array}$ & $\begin{array}{l}\text { LILACS, } \\
\text { BDENF }\end{array}$ & $\begin{array}{c}\text { Português } \\
2020\end{array}$ \\
\hline $\begin{array}{l}\text { Santos, K. F., \& } \\
\text { Barbosa , M. }\end{array}$ & COVID- 19 e a Odontologia na prática atual & SciELO & $\begin{array}{c}\text { Português } \\
2020\end{array}$ \\
\hline $\begin{array}{l}\text { Weiping, W., } \\
\text { Xiaoliang, L., Fuquan, } \\
\text { Z., Jie, Q., \& Ke, H. }\end{array}$ & $\begin{array}{l}\text { Radiation Therapy During the COVID-19 Pandemic: Experience from } \\
\text { Beijing, China }\end{array}$ & MEDLINE & $\begin{array}{c}\text { Inglês } \\
2020\end{array}$ \\
\hline $\begin{array}{l}\text { Sancanari, S. N., \& } \\
\text { Nogueira, J. M. R. }\end{array}$ & $\begin{array}{l}\text { A arquitetura laboratorial e a proteção dos profissionais de saúde em tempos } \\
\text { de COVID-19 }\end{array}$ & $\begin{array}{l}\text { LILACS } \\
\text { SciELO }\end{array}$ & $\begin{array}{l}\text { Português } \\
2020\end{array}$ \\
\hline Gaspar, G. S., et al. & $\begin{array}{l}\text { Characterization of dental surgeons of Pernambuco in the COVID-19 } \\
\text { pandemic context: preliminary data }\end{array}$ & SciELO & $\begin{array}{l}\text { Inglês } \\
2020\end{array}$ \\
\hline Assadi, M., et al. & $\begin{array}{l}\text { Key elements of preparedness for pandemic coronavirus disease } 2019 \\
\text { (COVID-19) in nuclear medicine units }\end{array}$ & MEDLINE & $\begin{array}{c}\text { Inglês } \\
2020\end{array}$ \\
\hline
\end{tabular}




\begin{tabular}{|c|c|c|c|}
\hline Santos, J. L. G., et al. & $\begin{array}{l}\text { Como os hospitais universitários estão enfrentando a pandemia de COVID- } \\
19 \text { no Brasil? / ¿Cómo están enfrentando la pandemia de COVID-19 los } \\
\text { hospitales universitarios en Brasil? / How are university hospitals coping } \\
\text { with the COVID-19 pandemic in Brazil }\end{array}$ & $\begin{array}{l}\text { LILACS, } \\
\text { BDENF }\end{array}$ & $\begin{array}{l}\text { Português } \\
2020\end{array}$ \\
\hline $\begin{array}{l}\text { Gomes, V. G. M., \& } \\
\text { Santos, P. C. P. }\end{array}$ & $\begin{array}{l}\text { Protocolo COVID-19 para higienização de profissionais de saúde no } \\
\text { domicílio / Covid-19 protocol for hygienizing healthcare professionals at } \\
\text { home }\end{array}$ & $\begin{array}{l}\text { LILACS, } \\
\text { BDENF }\end{array}$ & $\begin{array}{l}\text { Português } \\
2020\end{array}$ \\
\hline $\begin{array}{l}\text { Hermida, P. M. V., et } \\
\text { al. }\end{array}$ & $\begin{array}{l}\text { Cuidados à pessoa suspeita de COVID-19 com sinais de gravidade na } \\
\text { Atenção Primária à Saúde / Care of suspected person of covid-19 with severe } \\
\text { signs in primary health care }\end{array}$ & $\begin{array}{l}\text { LILACS, } \\
\text { BDENF }\end{array}$ & $\begin{array}{l}\text { Português } \\
2020\end{array}$ \\
\hline Siman, A. G., et al. & $\begin{array}{l}\text { Produção de máscaras cirúrgicas: estratégia no combate à covid-19 / } \\
\text { Producción de máscaras quirúrgicas: estrategia para combatir el covid-19 / } \\
\text { Production of surgical masks: strategy to combat covid-19 }\end{array}$ & $\begin{array}{l}\text { LILACS, } \\
\text { BDENF }\end{array}$ & $\begin{array}{l}\text { Português } \\
2020\end{array}$ \\
\hline $\begin{array}{l}\text { Medeiros, M. S., } \\
\text { Santos, H. L. F., } \\
\text { Barreto, J. O., Freire, } \\
\text { J. C. P., \& Dias- } \\
\text { Ribeiro, E. }\end{array}$ & $\begin{array}{l}\text { COVID-19 pandemic impacts to Dentistry / Impactos da pandemia causada } \\
\text { pela covid-19 na Odontologia }\end{array}$ & $\begin{array}{l}\text { LILACS, } \\
\text { BBO }\end{array}$ & $\begin{array}{c}\text { Inglês } \\
2020\end{array}$ \\
\hline
\end{tabular}

Fonte: Autores.

Analisando os 15 artigos selecionados para compor a revisão, observou-se prevalência do idioma português (53,3\%), seguido pelo inglês $(46,7 \%)$. Em relação aos países de origem dos artigos, verificou-se que 12 são provenientes da América do Sul (Brasil, 80\%) e 3 pertencem ao continente asiático (China, Índia e Irã, 20\%). No que se refere ao ano de publicação, constatouse a prevalência do ano de 2020 (93,3\%) em relação aos artigos publicados no ano de 2021 (6,7\%). No que tange a base de dados, mereceu destaque a LILACS, com 10 artigos (66,7\%), seguida pela MEDLINE com 3 (20\%) artigos, e SciELO com 2 (13,3\%) estudos apenas.

Assim, o conhecimento produzido pela literatura investigada foi sintetizado em duas categorias temáticas: I - "Medidas de Biossegurança adotadas na pandemia de COVID-19"; e II - "Impactos da COVID-19 na Biossegurança e na qualidade de vida dos profissionais da saúde".

\section{Categoria I - Medidas de Biossegurança adotadas na pandemia de COVID-19}

Os estudos que compõem a categoria I, ressaltam a importância da adoção de medidas de biossegurança, para a prevenção e controle da COVID-19, minimizando o risco de transmissão, durante o atendimento dos pacientes acometidos, assim como caracterizar as medidas mais utilizadas nesse contexto.

A utilização de EPI's como máscara N95, protetor facial, luvas, aventais e gorros, reflete na intensificação de barreiras físicas, que visam proteger a face, corpo, cabelo, braços e pés do trabalhador, de modo rigoroso, diminuindo a transmissibilidade da COVID-19, além da manutenção do cuidado com a higiene, e descarte adequado de resíduos contaminados (Misra et al., 2020; Vicente, Silva, \& Barbosa, 2020).

No que se refere a ambientes odontológicos, as medidas de biossegurança envolvem desde a detecção sintomática de pacientes, com a realização de aferição da temperatura, e adequação aos testes para COVID-19. Além disso, para proteção de todos os envolvidos, é recomendado minimizar e evitar procedimentos que produzem gotículas ou aerossóis, como ejetores de saliva, pois os mesmos acarretam em alto risco de contaminação (Santos \& Barbosa, 2020). 
No âmbito da radiologia, novas medidas de proteção contra o coronavírus são necessárias, para pacientes submetidos a radioterapia assim como para a equipe médica, recomenda-se a organização do departamento de Radioterapia em duas áreas: uma área limpa e uma área semi contaminada com os EPI's correspondentes usados nessas zonas, para minimizar o contágio e exposição. Para triagem dos pacientes, a temperatura dos mesmos e de seus acompanhantes deve ser aferida (Weiping, Xiaoliang, Fuquan, Jie, \& Ke, 2020).

Quanto à medicina nuclear, um modelo de gestão específico, em conjunto com medidas preventivas, para reduzir a probabilidade de contágio devem ser adotados pelos profissionais de saúde, de acordo com as recomendações nacionais. Além disso, ações de prevenção e cuidado são necessárias, como o uso de EPI's e a adoção de medidas de higiene, como a lavagem de mãos (Assadi et al., 2020).

Cuidados psicológicos também são necessários, visto que, na pandemia, ocorre o confinamento e isolamento de grande parte da população, então, nessa perspectiva, são necessários apoio psicológico e comportamental, além de novas atividades de lazer e atividade física, que aumentam os níveis de endorfina, evitando o estresse e ansiedade, que são comuns durante a pandemia (Assadi et al., 2020).

A literatura atenta para o cumprimento de protocolos a serem seguidos, a exemplo um protocolo sintetizado em checklist de 19 passos capazes de reduzir a transmissão do vírus SARS-CoV-2, seja em ambientes laborais dos profissionais de saúde ou em ambientes domiciliares (Gomes \& Santos, 2020). No tocante à Atenção Primária à Saúde, foi elaborado um checklist, a ser utilizado coletivamente pelos profissionais, proporcionando maior segurança no cuidado a pacientes com COVID-19, acesso rápido às informações, favorecendo o diálogo entre os profissionais durante o atendimento, a comunicação com a família e com outros pontos da Rede de Atenção às Urgências (Hermida et al., 2020).

Ademais, durante a pandemia, a utilização de EPI 's tornou-se cada vez mais necessária, o que repercutiu negativamente na disponibilidade desses insumos em diversos países, preocupando as autoridades sanitárias em nível mundial (Silveira et al., 2021).

Diante da escassez de EPIs disponíveis aos profissionais da saúde no cenário da COVID-19, estudo apontou estratégias que visam subsidiar tal contexto, como a produção de máscaras cirúrgicas, via produção de inovação tecnológica, com garantia de testes de qualidade, posteriormente distribuídas a diversos serviços de saúde (Siman et al., 2020).

\section{Categoria II - Impactos da COVID-19 no contexto da Biossegurança e na qualidade de vida dos profissionais da saúde}

A presente categoria evidenciou que é de inconteste necessidade o uso de Equipamento de Proteção Individual (EPI) adequado e de forma correta, todavia ainda não há consenso sobre quais são as mais eficazes nesse contexto (Godoi, Bernardes, Novais, Nogueira, \& Pinheiro, 2020).

Profissionais da saúde, como a exemplo, enfermeiros brasileiros têm enfrentado precarização no processo de trabalho, devido a diversos problemas no sistema de saúde, tais como: infraestrutura inadequada para o atendimento, escassez de insumos, falta de EPI's, sobrecarga de trabalho com jornadas extensas, baixos salários, o que culmina no comprometimento da qualidade de vida destes profissionais (Quadros, Fernandes, Araujo, \& Caregnato, 2020).

Infere-se também que é de extrema importância que profissionais expostos em ambientes como laboratórios, principalmente aqueles que desenvolvem pesquisas voltadas a COVID-19, devem atuar com disponibilidade de proteção biológica adequada, conforme normas de biossegurança, evitando comprometer sua saúde e qualidade de vida (Sancanari \& Nogueira, 2020).

É válido ressaltar a extrema exposição de profissionais como cirurgiões dentistas, reforçando-se que esta categoria se encontra imersa a riscos ocupacionais, cuja necessidade de treinamentos devem envolver desde as noções de biossegurança e cuidados específicos, no enfrentamento da COVID-19 (Gaspar et al., 2020). 
Desde o início da pandemia, alguns hospitais universitários adotaram medidas bruscas em setores de Assistência, Gestão, Extensão, Ensino e Pesquisa, havendo suspensão de atividades educativo-formativas, que permitiu o desenvolvimento de materiais educativos sobre medidas de prevenção da COVID-19. Além disso, foram suspensas consultas não relacionadas à COVID-19 e cirurgias eletivas, com contratação emergencial de profissionais da saúde e oferta de atendimento psicológico para os trabalhadores (Santos et al., 2020).

Apesar dos impactos negativos, a COVID-19 possibilitará futuramente diversos avanços tecnológicos e grandes inovações, como a integralização e adoção de medidas de biossegurança. Contudo, sua presença ainda prejudica em grau elevado o ambiente hospitalar, sendo o perigo maior voltado para os aerossóis durante os procedimentos odontológicos, necessitando impor medidas de biossegurança, tais como: redução dos atendimentos como medida mais eficaz, redução de equipamentos que resultam na liberação de gotículas, higienização do local durante o atendimento, realização da pré-triagem do paciente e a utilização necessária de EPI's em todos os procedimentos (Medeiros, Santos, Barreto, Freire, \& Dias-Ribeiro, 2020).

\section{Considerações Finais}

O estudo em questão apresentou uma caracterização da produção científica acerca das medidas de biossegurança no contexto da pandemia de Covid-19, em periódicos online, no período de junho de 2020 a março de 2021. Os resultados mostraram que após o estudo integral das obras que compuseram o acervo científico deste artigo, foi possível observar que a pandemia do COVID-19 trouxe mudanças significativas no modo de trabalho e na qualidade de vida dos profissionais de saúde. Tais mudanças estão atreladas a fatores como a falta inicial de manejo correto, direcionado ao SARS-CoV-2, assim como a necessidade de normas de biossegurança mais rígidas, a serem seguidas pelos profissionais de saúde.

Destarte, é fato que a pandemia acentuou problemas previamente existentes, como escassez de insumos, altas jornadas de trabalhos, adoecimento mental, falta de capacitações para retirada e colocada de EPI 's. Todos esses fatores aliados com a falta de conhecimento acerca do novo coronavírus, corroboraram para a morte de muitos profissionais.

Nessa panorâmica, observou-se uma atenção às questões sobre biossegurança, com a criação de novos protocolos de segurança, para conter a infecção pelo referido vírus, seguindo a utilização de EPIs. Ademais, um grande desafio apresentado foi a padronização do conjunto de medidas de proteção, ao passo que novos equipamentos começaram a ser utilizados e popularizados, destacando a máscara N95 e o Face Shield como os mais relevantes no acervo científico. Além disso, foram propostas medidas na tentativa de contenção da disseminação do vírus, como o estímulo à higiene pessoal, como a lavagem de mãos e o uso de álcool em gel.

Em suma, o estudo apresentado trouxe uma nova reflexão sobre o tema, para acrescentar à comunidade científica e facilitar o entendimento de uma temática nova, tão complexo e ainda pouco estudada. No Brasil, o número de publicações geradas acerca dessa temática ainda é escasso, portanto, este estudo trouxe subsídios para o profissional da área de saúde assimilar os conceitos inerentes às medidas de biossegurança no contexto atual, e atentar para a necessidade de que mais pesquisas sobre essa temática sejam realizadas, a fim de possibilitar novas reflexões sobre uma capacitação de boa qualidade dos profissionais da saúde.

\section{Referências}

Assadi, M., et al., (2020). Key elements of preparedness for pandemic coronavirus disease 2019 (COVID-19) in nuclear medicine units. Eur J Nucl Med Mol Imaging, 47(8), 1779-1786.

Campos, A. C. V., \& Leitão, L. P. C. (2021). Letalidade da COVID-19 entre profissionais de saúde no Pará, Brasil. Journal Health NPEPS, 6(1), 22-34.

Gallasch, C. H., Cunha, M. L., Pereira, L. A. S., \& SIlva-Junior, J. S. (2020). Prevenção relacionada à exposição ocupacional do profissional de saúde no cenário de COVID-19. Rev. enferm. UERJ, 28, e49596. 
Gaspar, G. S., et al., (2020). Characterization of dental surgeons of Pernambuco in the COVID-19 pandemic context: preliminary data. Pesqui. Bras. Odontopediatria Clín. Integr., 20(Suppl. 1), e0133.

Gautier, J. F., \& Ravussin, Y. A. (2020). New Symptom of COVID-19: Loss of Taste and Smell. Obesity, 28(5), 848-848.

Godoi, A. P. N., Bernardes, G. C. S., Novais, R. L. R., Nogueira, L. S., \& Pinheiro, M. B. (2020). Focus on Health professionals during the SARS-COV-2 pandemic- Literature Review. Rev. Rede cuid.saúde, 14(2), 43-52.

Gomes, V. G. M., \& Santos, P. C. P. (2020). Protocolo COVID-19 para higienização de profissionais de saúde no domicílio. Enferm. foco, 11(2), $126-133$.

Hermida, P. M. V., et al., (2020). Cuidados à pessoa suspeita de COVID-19 com sinais de gravidade na Atenção Primária à Saúde. Enferm. foco, 11(2), 192198.

Johns Hopkins University \& Medicine (2020). Coronavirus Resource Center. https://coronavirus.jhu.edu/map.html.

Medeiros, M. S., Santos, H. L. F., Barreto, J. O., Freire, J. C. P., \& Dias-Ribeiro, E. (2020). Impactos da pandemia causada pela covid-19 na Odontologia. Rev Gaúcha Odontol, 68, e20200021.

Misra, V., et al., (2020). Guidelines for various laboratory sections in view of COVID-19: Recommendations from the Indian Association of Pathologists and Microbiologists. Indian J Pathol Microbiol, 63(3), 350-357.

Organização Mundial de Saúde (OMS) (2021). Folha informativa sobre a COVID-19. https://news.un.org/en/story/2021/10/1103642.

Quadros, A., Fernandes, M. T. C., Araujo, B. R., \& Caregnato, R. C. A. (2020). Desafios da Enfermagem Brasileira no Combate da COVID-19: uma reflexão. Enferm. foco, 11(1), 78-83.

Ribeiro, A. P., Oliveira, G. L., Silva, L. S., \& Souza, E. R. (2020). Saúde e segurança de profissionais de saúde no atendimento a pacientes no contexto da pandemia de Covid-19: revisão de literatura. Rev. bas. saúde ocup., 45(25), 1-12.

Ribeiro, G., Pires, D. E. P., \& Scherer, M. D. A (2016). Práticas de biossegurança no ensino técnico de enfermagem. Trab. educ. saúde, 14(3), 871-888.

Sancanari, S. N., \& Nogueira, J. M. R. (2020). A arquitetura laboratorial e a proteção dos profissionais de saúde em tempos de COVID-19. Rev. bras. anal. clin, 52(2), 186-191.

Santos, K. F., \& Barbosa, M. (2020). COVID- 19 e a Odontologia na prática atual. Revista Eletrônica Acervo Saúde, 12 (11), e5113.

Santos, J. L. G., et al., (2020). Como os hospitais universitários estão enfrentando a pandemia de COVID-19 no Brasil? Acta Paul Enferm., 33 , 1-8.

Silveira, M. G. S. S., et al., (2021). Changes in dental practice in times of COVID-19: review and recommendations for dental health. Rev. Gaúch. Odontol, 69, e2021001.

Siman, A. G., et al., (2020). Produção de máscaras cirúrgicas: estratégia no combate à covid-19. Rev. baiana enferm, 34 , e37234.

Soares, S. S. S., et al., (2020). Pandemia de Covid-19 e o uso racional de equipamentos de proteção individual. Rev. enferm. UERJ, 28 , e50360.

Souza, M. T., Silva, M. D., \& Carvalho, R. (2010). Revisão integrativa: o que é e como fazer. Einstein, 8(1 pt 1), $102-106$.

Teixeira, P., \& Valle, S. (2010). Biossegurança: uma abordagem multidisciplinar (2a ed.). Fiocruz.

Ursi, E. S., \& Galvão, C. M. (2006). Prevenção de lesões de pele no perioperatório: revisão integrativa da literatura. Rev Latino-am Enfermagem, 14(1), 124131.

Vicente, K. M. S., Silva, B. M., \& Barbosa, D. N. (2020). Diretrizes de biossegurança para o atendimento odontológico durante a pandemia do COVID-19: revisão de literatura. Rev. Odont. de Araçatuba, 41(3), 29-32.

Weiping, W., Xiaoliang, L., Fuquan, Z., Jie, Q., \& Ke, H. (2020). Radiation Therapy During the COVID-19 Pandemic: Experience from Beijing, China. In Vivo, 34(Suppl. 3), 1675-1680. 